\title{
An eight-mRNA signature outperforms the IncRNA-based signature in predicting prognosis of patients with glioblastoma
}

\author{
Zhenyu Gong ${ }^{\dagger}$, Fan Hong ${ }^{\dagger}$, Hongxiang Wang, Xu Zhang and Juxiang Chen ${ }^{*}$ (D)
}

\begin{abstract}
Background: The prognosis of the glioblastoma (GBM) is dismal. This study aims to select an optimal RNA signature for prognostic prediction of GBM patients.

Methods: For the training set, the long non-coding RNA (IncRNA) and mRNA expression profiles of 151 patients were downloaded from the TCGA. Differentially expressed mRNAs (DEGs) and IncRNAs (DE-IncRNAs) were identified between good prognosis and bad prognosis patients. Optimal prognostic mRNAs and IncRNAs were selected respectively, by using univariate Cox proportional-hazards $(\mathrm{PH})$ regression model and LASSO Cox-PH model. Subsequently, four prognostic scoring models were built based on expression levels or expression status of the selected prognostic IncRNAs or mRNAs, separately. Each prognostic model was applied to the training set and an independent validation set. Function analysis was used to uncover the biological roles of these prognostic DEGs between different risk groups classified by the mRNA-based signature.
\end{abstract}

Results: We obtained 261 DEGs and 33 DE-IncRNAs between good prognosis and bad prognosis patients. A panel of eight mRNAs and a combination of ten IncRNAs were determined as predictive RNAs by LASSO Cox-PH model. Among the four prognostic scoring models using the eight-mRNA signature or the ten-IncRNA signature, the one based on the expression levels of the eight mRNAs showed the greatest predictive power. The DEGs between different risk groups using the eight prognostic mRNAs were functionally involved in calcium signaling pathway, neuroactive ligand-receptor interaction pathway, and Wnt signaling pathway.

Conclusion: The eight-mRNA signature has greater prognostic value than the ten-IncRNA-based signature for GBM patients based on bioinformatics analysis.

Keywords: Glioblastoma, mRNA, IncRNA, Prognosis, Risk score

\section{Background}

Glioblastoma (GBM) is the most aggressive primary brain neoplasm [1]. The treatment involves maximal surgical resection along with radiotherapy and chemotherapy [2]. However, the prognosis of GBM patients is dismal that they have a survival of only $12-15$ months

\footnotetext{
* Correspondence: juxiangchen@smmu.edu.cn

${ }^{\dagger}$ Zhenyu Gong and Fan Hong contributed equally to this work.

Department of Neurosurgery, Changzheng Hospital, Second Military Medical University, NO. 415 Fengyang Road, Huangpu Distinct, Shanghai 200003, China
}

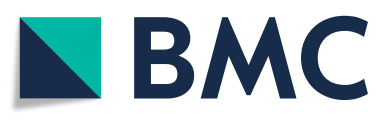

( The Author(s). 2020 Open Access This article is licensed under a Creative Commons Attribution 4.0 International License, which permits use, sharing, adaptation, distribution and reproduction in any medium or format, as long as you give appropriate credit to the original author(s) and the source, provide a link to the Creative Commons licence, and indicate if changes were made. The images or other third party material in this article are included in the article's Creative Commons licence, unless indicated otherwise in a credit line to the material. If material is not included in the article's Creative Commons licence and your intended use is not permitted by statutory regulation or exceeds the permitted use, you will need to obtain permission directly from the copyright holder. To view a copy of this licence, visit http://creativecommons.org/licenses/by/4.0/. The Creative Commons Public Domain Dedication waiver (http://creativecommons.org/publicdomain/zero/1.0/) applies to the data made available in this article, unless otherwise stated in a credit line to the data. after the standard treatment, with the 5-year survival rate of $3-5 \%[1,3]$. Therefore, it is important to develop therapeutic biomarkers for improving survival of GBM patients.

Considerable efforts have been made to identify prognostic gene signatures for GBM. For instance, a threegene signature of prognostic value for patients with MGMT promoter-methylated GBM is reported by performing bioinformatics analysis [4]. A study finds that a combination of Notch and hypoxia genes is closely 
associated with survival of GBM patients [5]. Silencing of the signal transducer and activator of transcription-3 (STAT3), an important mediator for the subtype of highly aggressive mesenchymal GBM, by a novel aptamer-siRNA chimera (Gint4.T) inhibits tumor growth and angiogenesis in a mouse model. Thus, Gint4.TSTAT3 is suggested as a novel molecule therapy for GBM [6]. In addition, the phosphorylation of STAT3 on Serine727 is identified as a potential prognostic marker for GBM patients [7].

Long non-coding RNAs (lncRNAs) have been focused recently because of its versatile roles in multiple biological processes [8]. Dysregulation of lncRNAs may be potential biomarkers and therapeutic targets for cancer [9]. A study investigates prognostic lncRNAs in GBM by constructing a functional GBM lncRNA-mediated ceRNA network [10]. Besides, using lncRNA expression profiles in GBM patients from The Cancer Genome Atlas (TCGA), a prognostic six-lncRNA signature is identified by applying survival analysis and Cox regression model [11]. Moreover, serum lncRNA HOTAIR is proposed as a prognostic biomarker for GBM [12]. Nevertheless, most of these studies have not validated the predictive accuracy of these prognostic signatures and the optimal prognostic model of GBM has not been established.

In the current study, we analyzed the expression of mRNAs and lncRNAs that related to the prognosis in GBM patients from TCGA and built a prognostic prediction model. In addition, accuracy of the predictive model was validated using data in the Chinese Glioma Genome Atlas (CGGA) database. Differential expression on mRNAs were analyzed between high- and low-risk groups based on the optimal prognostic model. Further function and pathway enrichment analyses were performed to provide hints concerning the roles of the prognostic genes in GBM development and physiology.

\section{Materials and methods \\ Data source}

The GBM RNA-seq data were downloaded from the TCGA portal (https://gdc-portal.nci. nih.gov/), containing 173 samples (154 primary GBM tissue samples, 14 recurrent samples and 5 normal controls) that sequenced on the platform of Illumina HiSeq 2000 RNA Sequencing (https://tcga.xenahubs.net/download/TCGA. GBM.sampleMap/HiSeqV2.gz). Among them, 151 primary GBM tissue samples that had survival and prognostic information were used as the training set (TCGA set). Meanwhile we downloaded RNA-seq data of 272 gliomas samples named "Part D" (http://cgga.org.cn/ download/20191128/CGGA.mRNA_array_301_gene_ level.20191128.txt.zip) [13] from the CGGA (http://cgga. org.cn/), 138 of which were histologically confirmed to be GBM, and were then used as the validation set. For the both two sets, the raw data of the Fragments Per Kilobase of exon model per Million mapped fragments (FPKM) in TXT format were downloaded, and then were normalized using the method as demonstrated in a previous study [14], to eliminate the deviation at expression level due to different sequencing platforms or diverse experiment backgrounds.

\section{Differentially expressed mRNAs and IncRNAs}

Briefly, according to RefSeq ID information provided by the training set and the validation set, we annotated mRNAs and lncRNAs of the two sets based on HUGO Gene Nomenclature Committee (HGNC) [15] (http:// www.genenames.org/), the database which records information of $4055 \operatorname{lncRNAs}$ and 19,198 protein-coding genes (https://www.genenames.org/cgi-bin/genegroup/ download-all). In the training set, samples were classified based on their clinical prognostic information. According to the sample characteristics, the good prognosis samples was defined as patients with overall survival (OS) of more than 6 months and was alive; whereas bad prognosis samples were those with OS less than 6 months and were died. Then, differentially expressed mRNAs (DEGs) and lncRNAs (DE-lncRNAs) were screened between good and bad prognosis patients using the limma package (version 3.34.7) of R. False discovery rate $(\mathrm{FDR})<0.05$ and $\mid \log _{2}$ fold change (FC) $\mid>1$ were set as the cutoff for significance. Twoway hierarchical clustering analysis based on centered Pearson correlation algorithm was carried out for these identified DEGs and DE-lncRNAs by pheatmap package [16] (version 1.0.8) of $R$.

\section{Prognosis prediction models}

In the training set, in order to develop prognosis prediction models, firstly, we determined prognosis-related lncRNAs and mRNAs that were significantly linked to OS from the identified DEGs and DE-lncRNAs by applying univariate Cox regression model and log-rank test. A gene or a lncRNA with $\log$-rank $p<0.05$ was considered as significant. These identified prognosis-related DEGs and DE-lncRNAs were then used to fit L1-penalized (LASSO) Cox-PH regression model [17] for selection of the optimal predictive mRNAs and lncRNAs with penalized package (version 0.9-50) of R (http://bioconductor. org/packages/penalized/). The optimal parameter of 'lambda' in the selection model was calculated via the cross-validation likelihood (cvl) method for 1000 times.

\section{Prognosis prediction models based on expression status of prognostic RNAs}

$\mathrm{X}$-Tile tool [18] was applied to calculate the optimal cutoff point for expression levels of the aforementioned 
prognostic mRNAs and lncRNAs based on patients' survival. Monte-Carlo $P<0.05$ were set as the threshold for the cutoff points. Then the RNA expression status of a sample was defined based on the RNA's cutoff value: if expression level of a RNA is above the cutoff value (high expression), its expression status is equal to 1; otherwise, the expression level below the cutoff value (low expression), the RNA expression status of is equal to 0 . Combing the expression status with the Cox regression coefficients of these prognostic RNAs, the sample risk assessment system was constructed, and the risk score of each sample was obtained using the following formula:

$$
\text { Status Risk Score }=\sum \beta_{\text {RNAn }} \times \text { Status }_{\text {RNAn }} .
$$

$\beta_{\text {RNAn }}$ indicates Cox-PH coefficient of a RNA; Status RNAn indicates expression status of a RNA.
Prognosis prediction model based on expression levels of prognostic RNAs

Using expression levels of the DEGs and DE-lncRNAs, and their Cox regression coefficients, the prognostic scoring models were established with the following formula:

$$
\text { Expression Risk Score }=\sum \beta_{\mathrm{RNAn}} \times \operatorname{Exp}_{\mathrm{RNAn}} .
$$

$\beta$ RNAn and Exp RNAn represents Cox-PH coefficient and expression level of a RNA, respectively. For lncRNA, the expression unit is FPKM.

To detect the predictive ability of different prognostic scoring models, they were tested on the training set and the validation set, separately. According to median risk score, each set was classified into a high-risk group and a low-risk group. Thereafter, Kaplan-Meier (KM) method was used to assess the survival curves between

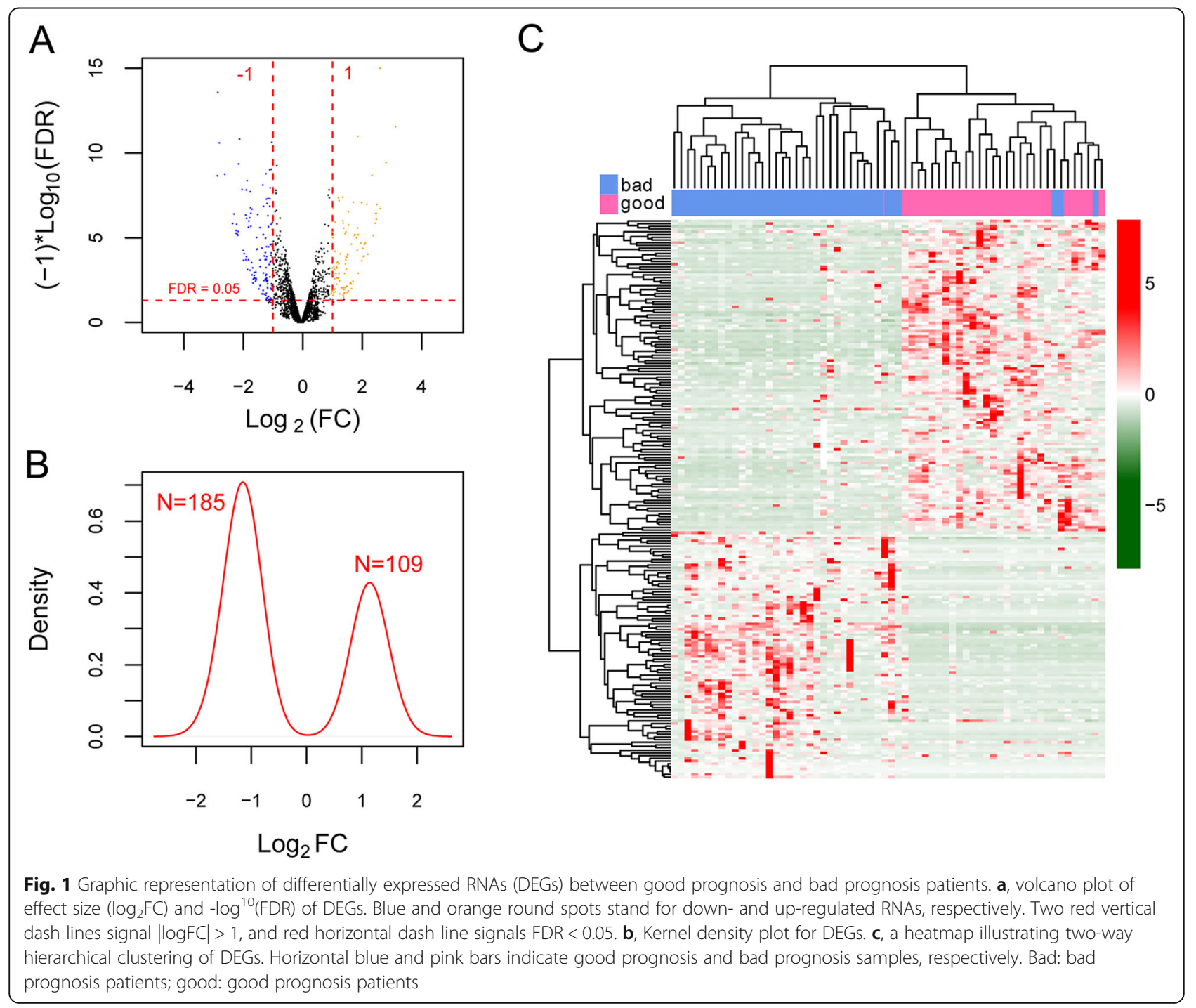


groups. Additionally, the accuracy for survival prediction using these models were evaluated by area of the receiver operating characteristic curve (AUC). By integrating the parameters of the four models in both training set and validation set, the optimal prognosis prediction model was selected.

\section{Nomogram survival rate model for independent prognostic factor}

Using the univariate and multivariate regression analysis in the survival package of $\mathrm{R}$ (version 2.41-1), the prognostic factors in the training set were selected with the significance threshold of log-rank $p<0.05$.

To further investigate the correlations between the independent prognostic factors and the survival, we combined the identified independent prognostic factors with the predicted risk information in the prediction prognosis model to construct a nomogram 1-year or 3-year survival rate model [19] by using rms package of $\mathrm{R}$. The actual and predicted probabilities of 1year OS and 3-year OS were compared using calibration plots. $P<0.05$ suggests statistical significance.

\section{Function analysis}

All samples in the training set were divided into high-risk and low-risk groups according to the risk score obtained from the optimal prognosis prediction model. Then, DEGs between the two risk groups were analyzed using the limma package in $R$ (version
3.34.7), with the cutoff of FDR $<0.05$ and $\left|\log _{2} \mathrm{FC}\right|>$ 0.263 . The FC here was set as 1.2 because if it was set as 2 as aforementioned, the DEG numbers were small, which would impede the following enrichment analysis.

Afterwards, using the clusterProfiler package [20] (version 3.6.0) of R, we performed gene ontology (GO) function and Kyoto Encyclopedia of Genes and Genomes (KEGG) pathway enrichment analyses to obtain the biological processes and KEGG signaling pathways that were significantly enriched with these identified DEGs. Statistical significance was set at $p<0.05$.

\section{Results}

\section{Identification of DEMs and DELs}

Following data annotation, we obtained 17,299 mRNAs and 770 lncRNAs overlapped by the TCGA set and the validation set. Based on predefined classification criteria, there identified 27 good prognosis samples and 37 bad prognosis samples in the TCGA set. In total, 261 mRNAs and 33 lncRNAs were differentially expressed between good prognosis and bad prognosis samples of the training set, including 182 down-regulated and 79 up-regulated mRNAs, 3 down-regulated and 30 upregulated lncRNAs. To show whether FDR value and $\left|\log _{2} \mathrm{FC}\right|$ conformed to logic with different test, we illustrated these results in a volcano plot (Fig. 1a) and a kernel density plot (Fig. 1b). Two-way hierarchical clustering of these DEGs and DE-lncRNAs was depicted in a

Table 1 Ten IncRNAs and eight mRNAs selected as prognostic RNAs

\begin{tabular}{|c|c|c|c|c|c|c|}
\hline Type & ID & coefficient & $P$-value & $\mathrm{HR}$ & $95 \% \mathrm{Cl}$ & X-tile cutoff \\
\hline \multirow[t]{10}{*}{ IncRNA } & HOXB-AS3 & 0.074 & 0.007 & 1.350 & $1.086-1.676$ & 0.87 \\
\hline & LGALS8-AS1 & 0.122 & 0.014 & 1.292 & $1.053-1.584$ & 0.20 \\
\hline & LINC00032 & -0.003 & 0.020 & 0.795 & $0.656-0.963$ & 0.20 \\
\hline & LINC00311 & 0.060 & 0.001 & 1.481 & $1.173-1.868$ & -2.01 \\
\hline & LINC00494 & -0.004 & 0.013 & 0.842 & $0.672-0.952$ & -0.14 \\
\hline & LINC00544 & 0.021 & 0.021 & 1.265 & $1.035-1.543$ & 0.02 \\
\hline & LINC00589 & 0.067 & 0.019 & 1.147 & $0.934-1.407$ & 0.00 \\
\hline & LINC00626 & -0.005 & 0.018 & 0.770 & 0.619-0.956 & 0.00 \\
\hline & MEIS1-AS3 & -0.053 & 0.040 & 0.803 & $0.650-0.989$ & 0.15 \\
\hline & VAV3-AS1 & 0.104 & 0.042 & 1.225 & $0.998-1.502$ & -0.09 \\
\hline \multirow[t]{8}{*}{ mRNA } & ASIC5 & 0.065 & 0.035 & 1.246 & $1.016-1.528$ & -1.67 \\
\hline & CGB7 & 0.072 & 0.003 & 1.405 & $1.124-1.756$ & -0.47 \\
\hline & CLDN16 & 0.123 & 0.006 & 1.372 & $1.096-1.717$ & -2.39 \\
\hline & HAPLN4 & 0.143 & 0.000 & 1.518 & $1.228-1.877$ & -2.03 \\
\hline & KRT13 & 0.084 & 0.002 & 1.362 & $1.123-1.652$ & -0.01 \\
\hline & LCN9 & 0.059 & 0.012 & 1.300 & $1.060-1.595$ & -0.22 \\
\hline & SOST & 0.033 & 0.018 & 1.148 & $1.038-1.404$ & 0.60 \\
\hline & TNS4 & 0.028 & 0.041 & 1.231 & $1.009-1.503$ & -0.14 \\
\hline
\end{tabular}


heatmap (Fig. 1c). The results showed that good prognosis samples could be well distinguished from bad prognosis samples based on expression patterns of these DEGs and DE-lncRNAs. The specific mRNA and IncRNA names are provided in the appendix 1.

\section{The prognostic model based on expression levels of 8} mRNAs was superior to the ten-IncRNA-based prognostic model

Combining the DEGs and DE-lncRNAs with the prognostic information, the univariate Cox regression analysis identified 21 of the 261 DEGs and 16 of the 33 DE-lncRNAs were significantly correlated with OS. These prognosis-related DEGs and DE-lncRNAs were then used to fit the LASSO Cox-PH regression model, respectively, to select the optimal RNA set relating to prognosis. As defined in the method section, the lambda value was determined by conducting $1000 \mathrm{cvl}$. A panel of 8 optimal DEG-set was obtained at the maximum value of cvl (-557.1395) when lambda was 7.1358; and 10 optimal DE-lncRNA set were obtained (lambda = 3.2821, cvlmax $=-558.6265) \quad($ Table 1). The eight mRNAs were acid sensing ion channel subunit family member $(A S I C)$, chorionic gonadotropin subunit beta $(C G B)$, claudin $(C L D N) 16$, keratin $(K R T) 13$, hyaluronan and proteoglycan link protein (HAPLN)4, lipocalin $(L C N)$ 9, sclerostin (SOST), and tensin (TNS)4. The ten lncRNAs consisted of homeobox (HOXB)-antisense (AS)3 (HOXB-AS3), LGALS8-AS1, Long intergenic nonprotein coding (LINC)00032 (LINC00032), LINC00311, LINC00494, LINC00544, LINC00589, LINC00626, MEIS1-AS3 and VAV3-AS1.

As aforementioned, X-Tile bio-informatics tool was used to explore the correlations between the above optimal sets with the prognosis, and to get the cutoff value of each RNA. After obtaining the expression status in each sample, a prognosis risk prediction model based on expression status and Cox-PH

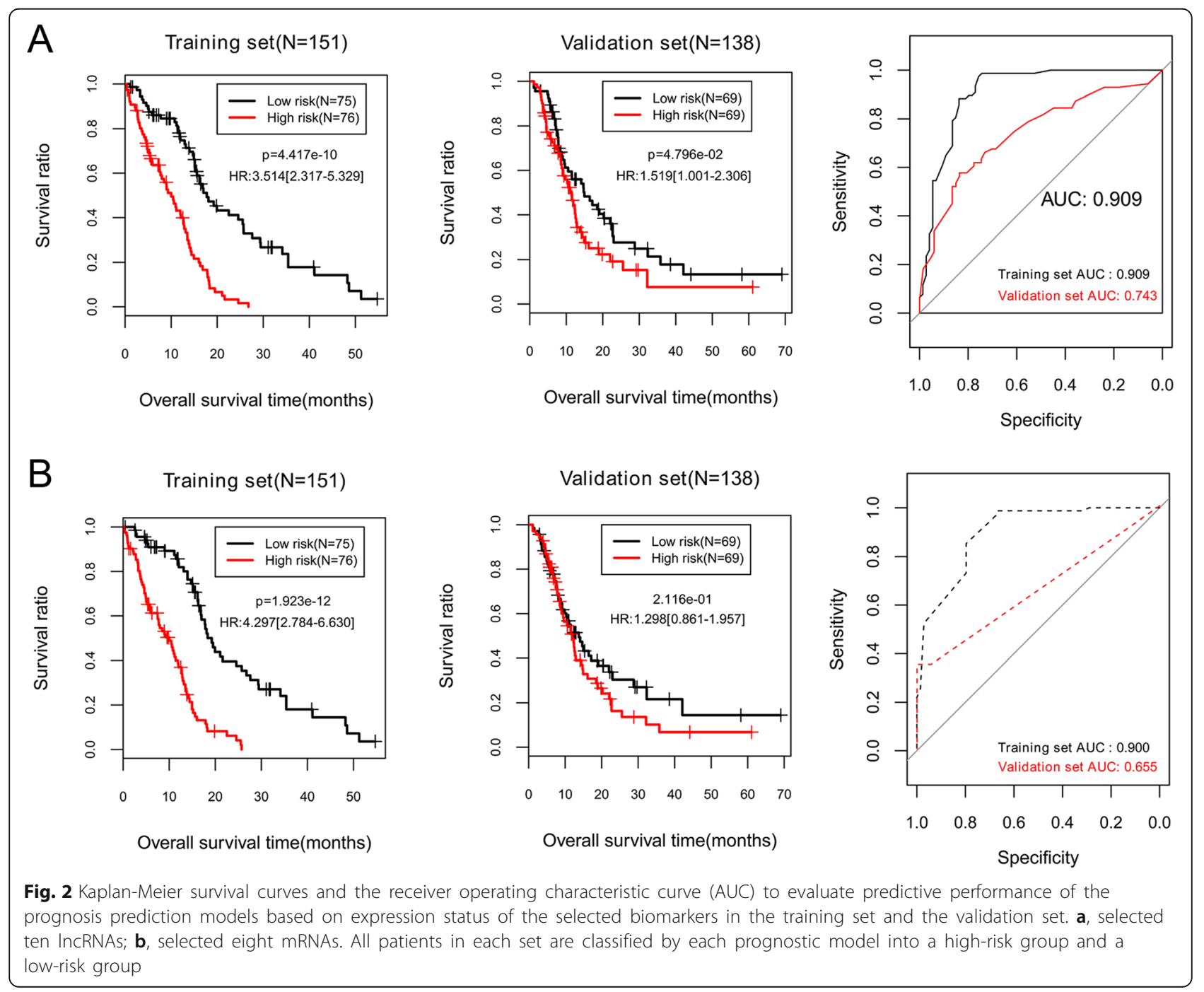




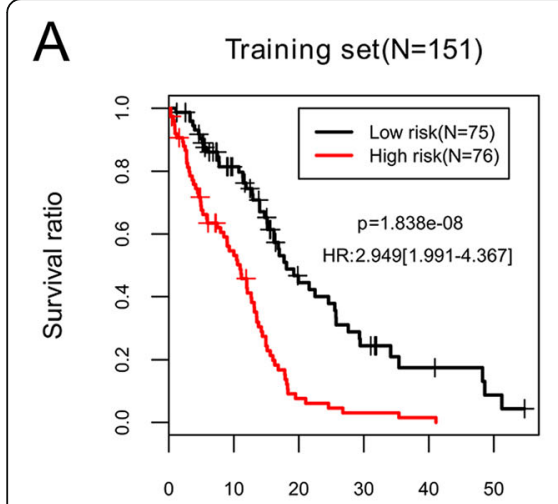

Overall survival time(months)

B

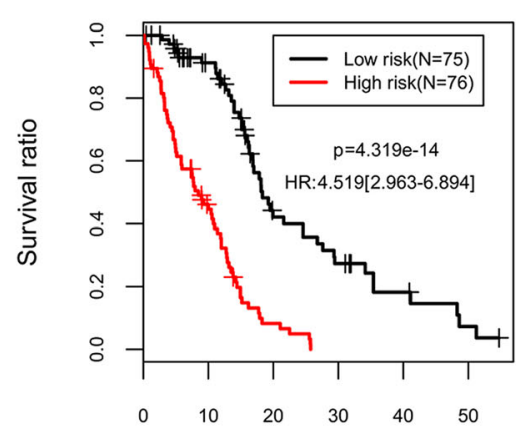

Overall survival time(months)

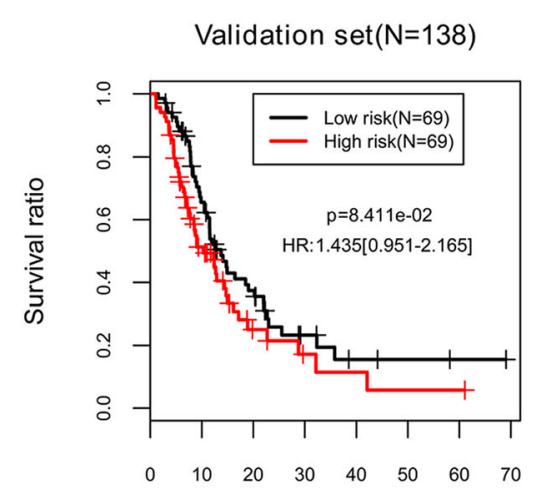

Overall survival time(months)

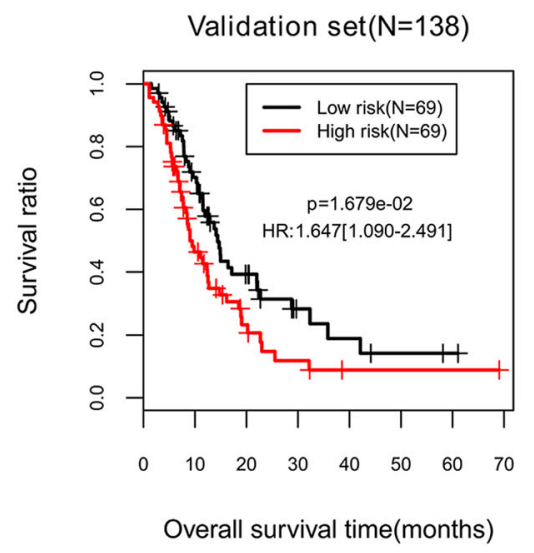

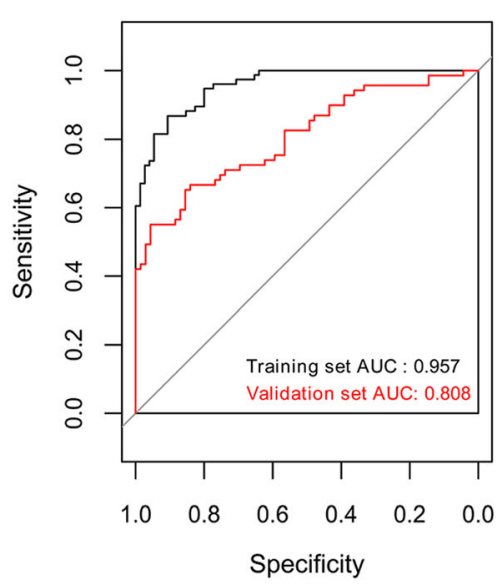

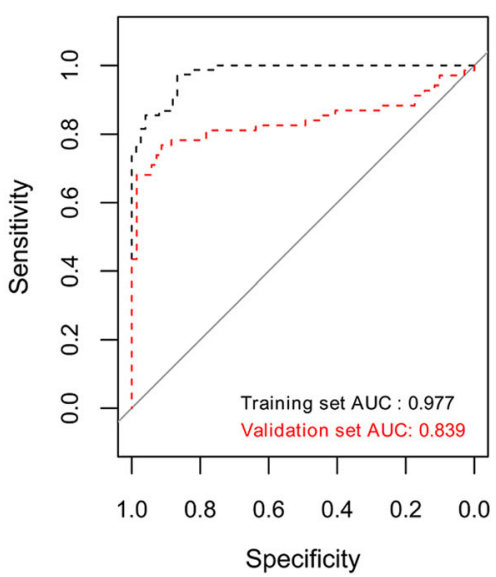

Fig. 3 Kaplan-Meier survival curves and the receiver operating characteristic curve (AUC) to assess predictive ability of the prognosis prediction models based on expression levels of the selected biomarkers in the training set and the validation set. $\mathbf{a}$, selected ten IncRNAs; $\mathbf{b}$, selected eight mRNAs. Each set is separated by each prognostic model into a high-risk group and a low-risk group

coefficients of the ten prognostic lncRNAs was established as follows (model 1):

lncRNA status Risk Score $=(0.074) *$ Status $_{\mathrm{HOXB}-\mathrm{AS} 3}+$ $(0.122) *$ Status $_{\text {LGALS8-AS1 }}+(-0.003) *$ Status $_{\text {LINC00032 }}+$ $(0.060) *$ Status $_{\text {LINC00311 }}+(-0.004) *$ Status $_{\text {LINC00494 }}+$ $(0.021) *$ Status $_{\text {LINC00544 }}+(0.067) *$ Status $_{\text {LINC00589 }}+(-$ $0.005) *$ Status $_{\text {LINC00626 }}+(-0.053) *$ Status $_{\text {MEIS1-AS3 }}+$ $(0.104) *$ Status vav3- AS1.

The prognostic prediction model based on the eight prognostic mRNAs was developed as follows (model 2):
mRNA status Risk Score $=(0.065) *$ Status $_{\text {ASIC5 }}+$ $(0.072) *$ Status $_{\text {CGB7 }}+(0.123) *$ Status $_{\text {CLDN16 }}+(0.143) *$ Status $_{\text {HAPLN } 4}+(0.084) \quad * \quad$ Status $_{\text {KRT13 }}+(0.123) *$ Status $_{\mathrm{LCN} 9}+(0.143) *$ Status $_{\mathrm{SOST}}+(0.084) *$ Status TNS4.

The model based on Cox-PH coefficients and expression levels of the eight prognostic mRNAs were built as follows (model 3):

lncRNA Expression Risk Score $=(0.074) *$ Exp HOX- $_{\text {- }}$ B-AS3 $+(0.122) \quad * \quad$ Exp $_{\text {LGALS8-AS1 }}+(-0.003)$

$\operatorname{Exp}_{\text {LINC00032 }}+(0.060) * \operatorname{Exp}_{\text {LINC00311 }}+(-0.004)$

Table 2 Predictive performances of four prognosis prediction models based on expression levels or status of IncRNAs or mRNAs

\begin{tabular}{|c|c|c|c|c|c|c|c|c|}
\hline \multirow[t]{3}{*}{ Type } & \multicolumn{4}{|l|}{ Status model } & \multicolumn{4}{|c|}{ Expression model } \\
\hline & \multicolumn{2}{|c|}{ LogRank p-value } & \multicolumn{2}{|l|}{ AUC value } & \multicolumn{2}{|c|}{ LogRank p-value } & \multicolumn{2}{|l|}{ AUC value } \\
\hline & Training set & Validation set & Training set & Validation set & Training set & Validation set & Training set & Validation set \\
\hline IncRNA & $4.42 \times 10^{-10}$ & 0.0480 & 0.907 & 0.743 & $1.84 \times 10^{-08}$ & 0.0841 & 0.957 & 0.808 \\
\hline mRNA & $1.92 \times 10^{-12}$ & 0.2116 & 0.900 & 0.655 & $4.32 \times 10^{-14}$ & 0.0168 & 0.977 & 0.839 \\
\hline
\end{tabular}


Table 3 Determination of prognostic clinical factors

\begin{tabular}{|c|c|c|c|c|c|c|c|}
\hline \multirow[t]{2}{*}{ Clinical characteristics } & \multirow{2}{*}{$\begin{array}{l}\text { Training set } \\
(N=151)\end{array}$} & \multicolumn{3}{|c|}{ Uni-variables cox } & \multicolumn{3}{|c|}{ Multi-variables cox } \\
\hline & & $\mathrm{HR}$ & $95 \% \mathrm{Cl}$ & P-value & $\mathrm{HR}$ & $95 \% \mathrm{Cl}$ & $P$-value \\
\hline Age (years, mean $\pm S D$ ) & $59.82 \pm 13.67$ & 1.029 & $1.014-1.045$ & $1.966 \times 10^{-04}$ & 1.022 & $1.005-1.039$ & $1.310 \times 10^{-02}$ \\
\hline Gender (Male/Female) & $97 / 54$ & 0.899 & $0.613-1.320$ & $5.886 \times 10^{-01}$ & - & - & - \\
\hline Chemo-therapy (Yes/No/-) & $45 / 89 / 17$ & 0.603 & $0.397-0.915$ & $1.625 \times 10^{-02}$ & 1.791 & $0.895-3.585$ & $9.960 \times 10^{-02}$ \\
\hline Drug-therapy (Yes/No/-) & 20/113/18 & 0.729 & $0.431-1.234$ & $2.377 \times 10^{-01}$ & - & - & - \\
\hline Immuno-therapy (Yes/No/-) & 2/131/18 & 0.555 & $0.136-2.264$ & $4.050 \times 10^{-01}$ & - & - & - \\
\hline Pharmaceutical-therapy (Yes/No/-) & $55 / 82 / 14$ & 0.419 & $0.276-0.639$ & $3.239 \times 10^{-05}$ & 0.335 & $0.169-0.663$ & $1.700 \times 10^{-03}$ \\
\hline Targeted molecular-therapy (Yes/No/-) & 18/115/18 & 0.889 & $0.519-1.523$ & $6.701 \times 10^{-01}$ & - & - & - \\
\hline Radiotherapy (Yes/No/-) & 20/117/14 & 0.611 & $0.346-1.078$ & $8.575 \times 10^{-02}$ & - & - & - \\
\hline Risk status (High/Low) & $75 / 76$ & 4.519 & $2.963-6.894$ & $4.319 \times 10^{-14}$ & 4.179 & $2.631-6.640$ & $1.390 \times 10^{-09}$ \\
\hline Dead (Death/Alive/-) & $115 / 36$ & - & - & - & - & - & - \\
\hline Overall survival time (months, mean $\pm S D$ ) & $13.26 \pm 10.82$ & - & - & - & - & - & - \\
\hline
\end{tabular}

SD Standard deviation, $H R$ Hazard ratio, Cl Confidential interval

$\operatorname{Exp}_{\text {LINC00494 }}+(0.021) \quad * \quad \operatorname{Exp}_{\text {LINC00544 }}+(0.067)$

$\left.\operatorname{Exp}_{\mathrm{LINC} 00589}+(-0.005) * \operatorname{Exp}_{\mathrm{LINC}}\right) 0626+(-0.053)$ *

Exp $_{\text {MEIS1-AS3 }}+(0.104) *$ Exp $_{\text {VAV3-AS1 }}$.

Similarly, the model based on the ten lncRNAs was established as follows (model 4):

mRNA Expression Risk Score $=(0.065){ }^{*} \operatorname{Exp}_{\text {ASIC5 }}+$ $(0.072) * \operatorname{Exp}_{\mathrm{CGB} 7}+(0.123) * \operatorname{Exp}_{\mathrm{CLDN} 16}+(0.143) *$ Exp $_{\text {HAPLN } 4}+(0.084) * \operatorname{Exp}_{\text {KRT13 }}+(0.123) * \operatorname{Exp}_{\mathrm{LCN} 9}+$ $(0.143) * \operatorname{Exp}_{\text {SOST }}+(0.084) *$ Exp TNS4 $^{\text {. }}$

To compare prognostic performances of the four prognosis prediction models, all patients in the training set or the validation set were separated into a high-risk group and a low-risk group by risk score obtained from each model, separately (Figs. 2-3). OS of the two risk groups were analyzed using KM plot and log-rank test, and predictive ability of each model was measured by AUC curves. Log-rank $\mathrm{p}$ and AUC values were summarized in Table 2.

Among the four prognostic models, the model based on expression levels of the eight mRNAs generated the lowest $p$-values (training set, $p=4.32 \times 10^{-14}$; validation set, $p=0.01679$ ) and the highest AUC values (training set, $\mathrm{AUC}=0.977$; validation set, $\mathrm{AUC}=0.839$ ) for the

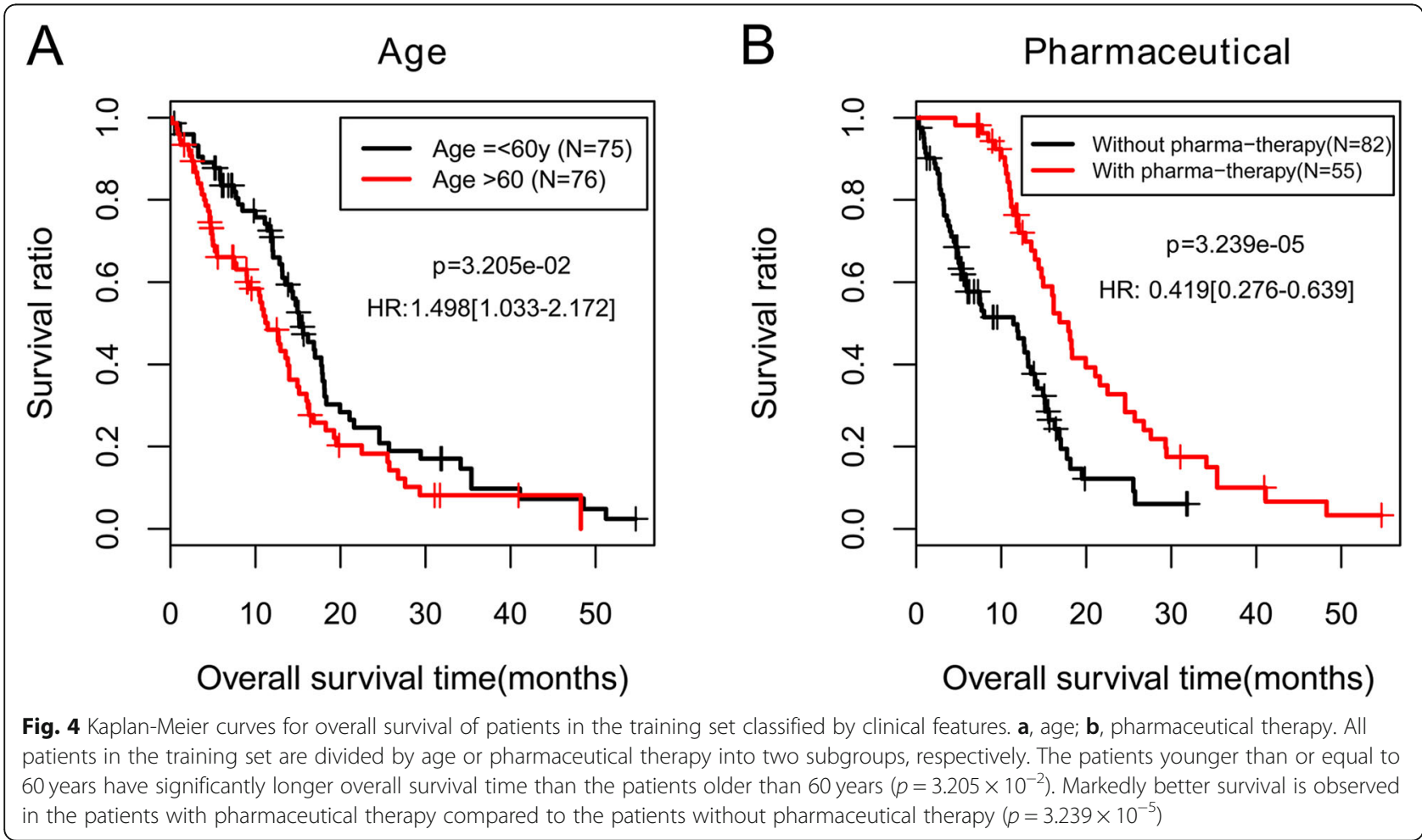


training set and the validation set (Table 2, Fig. 3b). These results indicate a better predictive capability of this prognostic model (model 3) than others, which was then selected for further analyses.

\section{Building nomogram based on prognostic clinical factors and the eight-mRNA signature}

As shown in Table 3 and Fig. 4, we found that age and pharmaceutical therapy were independent prognostic factors in the training set. Combining expression risk score based on the eight-mRNA signature with age and pharmaceutical therapy, we constructed a nomogram to improve predictive accuracy (Fig. 5a). As shown in calibration plots (Fig. 5b), consist results were observed between predicted and actual 1- and 3-year OS, respectively.

\section{Function analysis of the prognostic eight-gene signature}

We used the expression risk score of the eight-mRNAs in the mRNA expression based model (model 3) to classify samples in the TCGA set into high-risk group and low-risk group. We then screened DEGs between the two risk groups. As a result, 11 down-regulated and 255 up-regulated genes (selection criteria: FDR $<0.05$ and $|\log 2 \mathrm{FC}|>0.263$ ) were found between groups (Fig. 6). Detailed genes are provided in appendix 2 . These genes

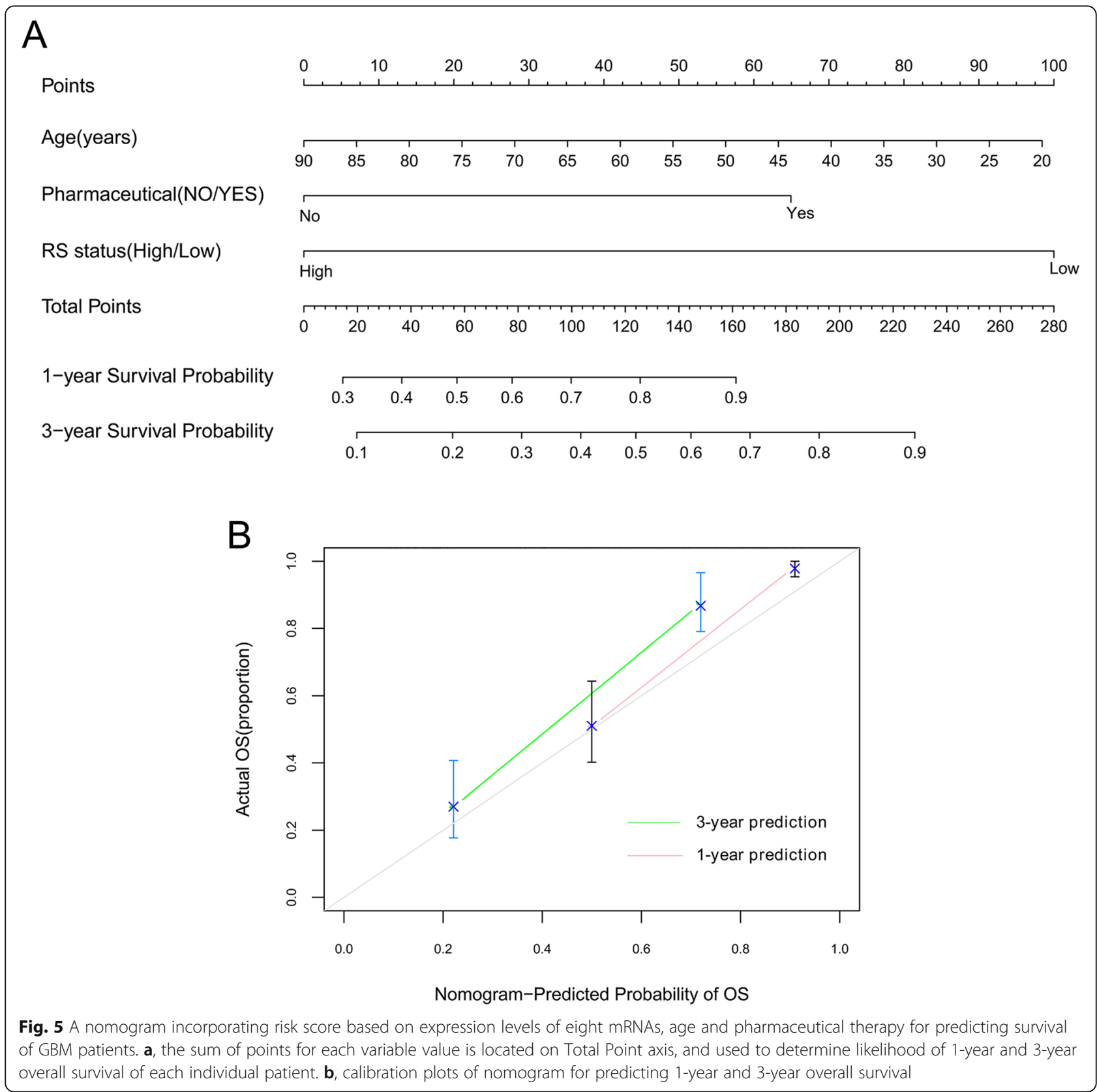




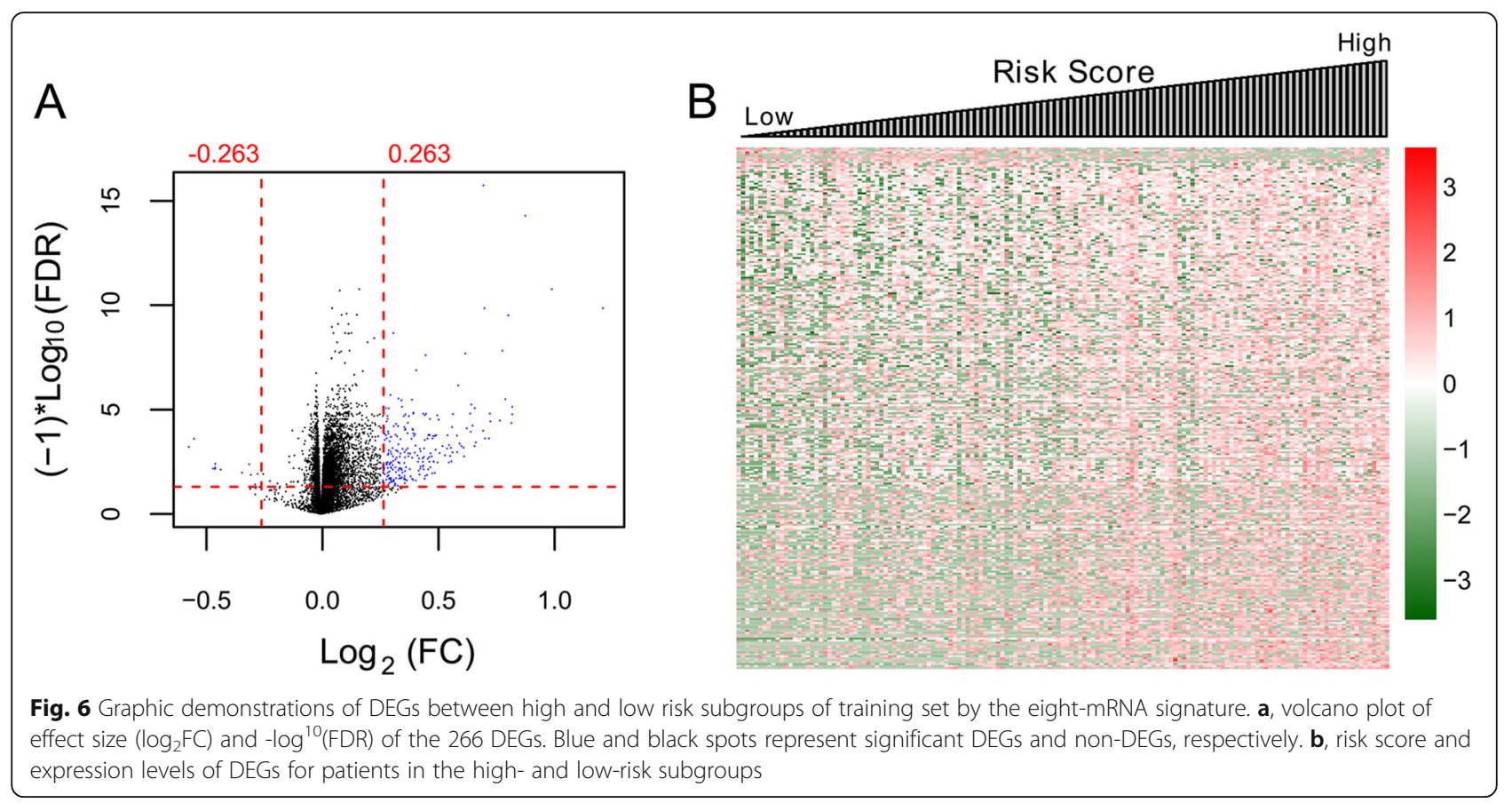

were significantly related to $25 \mathrm{GO}$ biological processes, such as cellular metal ion homeostasis and cellular calcium ion homeostasis (Table 4). Moreover, 5 KEGG signaling pathways functionally involved these DEGs, consisting of neuroactive ligand-receptor interaction, tight junction, Wnt signaling pathway, calcium signaling pathway, and cytokine-cytokine receptor interaction (Table 4).

\section{Discussion}

Prognosis of GBM patients varies greatly due to heterogeneity of the disease, demanding development of prognostic molecular indicators to improve outcome of GBM patients [21]. In the present study, we identified a ten-lncRNA signature and an eight-mRNA prognostic signature (ASIC5, CGB7, CLDN16, HAPLN4, KRT13, LCN9, SOST and TNS4) of GBM. Then, based on these signature sets, four prognosis prediction models were built using their expression levels or expression status, and the prognostic model based on expression levels of the eight- mRNA was superior to the others in predicting survival of GBM patients. To our knowledge, this is the first study that compares mRNA and lncRNA signatures for predicting outcome of GBM patients. The eight-mRNA signature was capable to classify GBM patients into subgroups with significantly different OS, showing greater prognostic power. Results of validation set revealed good reproducibility and robustness of the eight-mRNA signature. Application of this eightmRNA signature would help clinicians to select patients at high risk of death, thereby facilitating development of individualized therapies for GBM patients.

Of the eight mRNAs, ASIC5 is a member of ASIC channels that primarily locate in the central and peripheral nervous system. ASIC channels participate in diverse processes during tumor development, such as apoptosis, proliferation and migration, and may be potential therapeutic targets for cancer therapies [22]. Importantly, ASICs are correlated with malignant gliomas. ASIC1a and ASIC3 are expressed in GBM stem cell lines [23], and knockdown of ASIC1 in vitro inhibits the cell mobility and migration of GBM [24]. However, function of ASIC5 has not been revealed. Based on our study, expression of ASIC5 might be associated with prognosis of GBM.

CGB7 gene encodes the beta 7 subunit of chorionic gonadotropin. It has been demonstrated that human chorionic gonadotropin $\beta$ promotes development of GBM [25]. However, there are few studies reporting the correlation of this gene with prognosis. Our study might provide novel insights into GBM prognosis with this gene.

Claudin-16 encoded by CLDN16 is a component of tight junction strands. Abnormal tight junction functions have been established as a hallmark of human carcinomas [26]. Reduced expressions of CLDN1 and CLDN5, two family members of CLDN16, are associated with the progression of GBM multiforme [27]. According to our results, altered expression of CLDN16 might be also related to the development of 
Table 4 Significant GO biological processes and KEGG pathways

\begin{tabular}{|c|c|c|c|}
\hline Category & Term & Count of genes & $P$-value \\
\hline \multirow[t]{25}{*}{ Biology Process } & Cellular metal ion homeostasis & 12 & $6.570 \times 10^{-05}$ \\
\hline & Metal ion homeostasis & 12 & $9.840 \times 10^{-05}$ \\
\hline & Cellular cation homeostasis & 13 & $1.590 \times 10^{-04}$ \\
\hline & Cellular calcium ion homeostasis & 11 & $1.760 \times 10^{-04}$ \\
\hline & Calcium ion homeostasis & 11 & $2.200 \times 10^{-04}$ \\
\hline & Cellular di-, tri-valent inorganic cation homeostasis & 12 & $2.410 \times 10^{-04}$ \\
\hline & Di-, tri-valent inorganic cation homeostasis & 12 & $3.740 \times 10^{-04}$ \\
\hline & Cell-cell signaling & 20 & $4.350 \times 10^{-04}$ \\
\hline & Cation homeostasis & 13 & $4.710 \times 10^{-04}$ \\
\hline & Regulation of system process & 13 & $9.290 \times 10^{-04}$ \\
\hline & Ectoderm development & 10 & $1.434 \times 10^{-03}$ \\
\hline & Cellular ion homeostasis & 13 & $4.521 \times 10^{-03}$ \\
\hline & Cellular chemical homeostasis & 13 & $5.122 \times 10^{-03}$ \\
\hline & Chemical homeostasis & 15 & $8.944 \times 10^{-03}$ \\
\hline & Ion homeostasis & 13 & $8.989 \times 10^{-03}$ \\
\hline & Response to wounding & 15 & $1.186 \times 10^{-02}$ \\
\hline & Cellular homeostasis & 13 & $2.288 \times 10^{-02}$ \\
\hline & Behavior & 13 & $2.390 \times 10^{-02}$ \\
\hline & Multicellular organism reproduction & 13 & $3.075 \times 10^{-02}$ \\
\hline & Reproductive process in a multicellular organism & 13 & $3.075 \times 10^{-02}$ \\
\hline & Defense response & 15 & $3.669 \times 10^{-02}$ \\
\hline & Gamete generation & 11 & $3.984 \times 10^{-02}$ \\
\hline & Homeostatic process & 17 & $4.436 \times 10^{-02}$ \\
\hline & G-protein coupled receptor protein signaling pathway & 23 & $4.513 \times 10^{-02}$ \\
\hline & Transmission of nerve impulse & 10 & $4.589 \times 10^{-02}$ \\
\hline \multirow[t]{5}{*}{ KEGG Pathway } & Neuroactive ligand-receptor interaction & 9 & $7.151 \times 10^{-04}$ \\
\hline & Tight junction & 4 & $1.892 \times 10^{-02}$ \\
\hline & Wnt signaling pathway & 4 & $2.391 \times 10^{-02}$ \\
\hline & Calcium signaling pathway & 4 & $3.157 \times 10^{-02}$ \\
\hline & Cytokine-cytokine receptor interaction & 5 & $3.380 \times 10^{-02}$ \\
\hline
\end{tabular}

Count of genes, the total number of genes significantly involved in a GO biological process or pathway; GO, gene ontology; KEGG, Kyoto Encyclopedia of Genes and Genomes pathway enrichment

GBM, and the dysregulation might indicate prognosis of the disease.

HAPLNs are involved in forming and controlling perineuronal matrix in the adult brain, thus regulating neuronal function and plasticity [28]. HAPLN4 is obviously decreased in the parenchyma of malignant gliomas, and HAPLN4 expression promotes glioma cell adhesion and migration [29]. KRT13 encodes keratin 13 that is a type I cytokeratin (CK), and it is also named as CX13. Reportedly, $4 \%$ of GBM patients are estimated to be immunoreactive for cytokeratin, the reaction, however, is normally weak [30]. Although there is evidence that expressions of other CK members including CK34BE12, CK5, CK6, CK7, CK8, CK14, CK18, CK19 and CK20 are absent in GBM [31], there are rare reports on the roles of CK13 in GBM. Sclerostin encoded by SOST is produced primarily by osteocyte, and may be implicated in promoting tumor growth, bone metastasis and osteolysis in breast cancer [32]. Sclerostin is emerging as a potential target to treat cancer-related bone diseases [33]. Tensins localized in the cytoplasmic tails of integrins at focal adhesions are critical for cell adhesion, migration and invasion [34]. TNS4 plays an important part in stability of receptor tyrosine kinase, thus regulating survival and proliferation of carcinoma cells [35]. High expression of TNS4 is associated with a poor prognosis in many cancer types, such as gastric cancer and lung adenocarcinoma [36, 37]. However, studies on this gene 
in GBM have not been reported. Lipocalins are small extracellular proteins that participate in cell regulation, differentiation and proliferation [38]. Downregulation of LCN2 involves in the chemoresistance in GBM cells [39]. However, studies on the role of LCN9 in cancers are rare.. Our results might provide a hint for the future studies on the above genes. To gain insights into the functional roles of the eight prognostic mRNAs in the molecular mechanisms of GBM, GO function and KEGG pathway enrichment analyses were carried out for the DEGs between the predicted high-risk and lowrisk groups of the training set by the eight-mRNA signature. The results suggested that these genes were functionally associated with cellular metal ion homeostasis, cellular calcium ion homeostasis, calcium signaling pathway, neuroactive ligand-receptor interaction pathway, and Wnt signaling pathway. Increasing evidence has indicated that neuroactive ligand-receptor interaction pathway may play an important role in the biology of GBM [40, 41]. Oncogenic roles of Wnt signaling pathway in GBM has been demonstrated previously, and the pathway is also involved in GBM stem cell maintenance and invasion [42]. Calcium signaling is closely related to tumorigenesis and progression of GBM [43]. These findings collectively suggest the eight genes might be important regulators in GBM progression and might function via the involvement of the above processes and pathways. Further functional characterization of the eight-mRNA signature would be beneficial to unraveling the underlying mechanism of GBM.

LncRNAs are research hotspots currently, and several lncRNAs are identified as potential prognostic factors in GBM or glioma, such as TP73-AS1 [44], LINC00599 [45] and HOXA11-AS [46]. However, due to heterogeneity of the disease, it is insufficient to use a single lncRNA biomarker. A panel of ten-lncRNA signature relating to prognosis was identified in our study. Among them, LGALS8 is reported to promote cell proliferation in a GBM model [47]; VAV3 is highly expressed in breast cancer and GBM, and the overexpression indicates a poor prognosis of breast cancer [48]; LINC00311 is overexpressed in ankylosing spondylitis and promotes the proliferation and differentiation of osteoclasts [49, 50]. The remaining lncRNAs are rarely reported in previous studies. Our findings using them as a whole panel might provide novel insights into prognostic prediction of GBM. Interestingly, based on our study, the performance of the predictive prognosis with the ten-lncRNA signature was inferior to that using the eight-mRNA signature.

Although we have identified novel signatures for the prediction of GBM prognosis, and validated the accuracy using these predictive models in the validation set, several limitations remained in the study. First, the sample size was small in both of the training set and validation set, making a relatively weak statistic power. In addition, the prognostic value of these signatures needs to be validated in the GBM patients. Nevertheless, our study has predictive values and provides a foundation for future studies.

\section{Conclusion}

We develop an eight-mRNA and a ten-lncRNA prognostic signatures that are able to classify GBM patients into subgroups with different prognostic risks. The eightmRNA signature is superior to the ten-lncRNAs signature for prognostic risk classification of GBM patients. These findings would aid in improving outcome of GBM patients. Further validation of this eight-mRNA signature on large cohorts of GBM patients is warranted prior to clinical application.

\section{Supplementary information}

Supplementary information accompanies this paper at https://doi.org/10. 1186/s12881-020-0992-7.

Additional file 1.

Additional file 2.

\section{Abbreviations}

GBM: Glioblastoma; DEGs: Differentially expressed mRNAs; DEIncRNAs: IncRNAs; PH: Proportional-hazards; IncRNAs: Long non-coding RNAs; TCGA: The Cancer Genome Atlas; ROC: Receiver operating characteristic; GO: Gene ontology; CGB: Chorionic gonadotropin subunit beta

\section{Acknowledgements}

None.

\section{Authors' contributions}

ZG and FH performed data analyses and wrote the manuscript. HW and XZ contributed significantly in data analyses and manuscript revision. JC conceived and designed the study. All authors read and approved the final manuscript.

\section{Funding}

None.

\section{Availability of data and materials}

Raw data in this study were from the public databases, RNA sequencing data of 173 samples (154 primary GBM tissue samples, 14 recurrent samples and 5 normal controls) that sequenced on the platform of Illumina HiSeq 2000 RNA Sequencing (https://tcga.xenahubs.net/download/TCGA.GBM. sampleMap/HiSeqV2.gz) were from TCGA (https://gdc-portal.nci. nih.gov/) database; the RNA-seq data of 272 gliomas samples named "Part D" (http:// cgga.org.cn/download/20191128/CGGA.mRNA_array_301_gene_level.201 91128.txt.zip) were extracted from CGGA (http://cgga.org.cn/) database, and protein annotation information was from HGNC (http://www.genenames. org/) database; , and the annotation files were also downloaded (https:// www.genenames.org/cgi-bin/genegroup/download-all).

Ethics approval and consent to participate Not applicable.

Consent for publication

Not applicable.

Competing interests

The authors declare that they have no competing interests. 
Received: 19 June 2019 Accepted: 4 March 2020

Published online: 19 March 2020

\section{References}

1. Mcguire S. World Cancer Report 2014. Geneva, Switzerland: World Health Organization, International Agency for Research on Cancer, WHO Press, 2015. Adv Nutr. 2016;7(2):418.

2. Young RM, Jamshidi A, Davis G, Sherman JH. Current trends in the surgical management and treatment of adult glioblastoma. Ann Transl Med. 2015; 3(9):121.

3. Szopa W, Burley TA, Kramer-Marek G, Kaspera W. Diagnostic and Therapeutic Biomarkers in Glioblastoma: Current Status and Future Perspectives. BioMed Research Int. 2017;2017(2):8013575 2017,(2017-02-20).

4. Wang W, Zhang L, Wang Z, Yang F, Wang H, Liang T, Wu F, Lan Q, Wang J, Zhao J. A three-gene signature for prognosis in patients with MGMT promoter-methylated glioblastoma. Oncotarget. 2016;7(43):69991-9.

5. Khushboo I, Saroj Kant M, Chitrangda S, Harshit G, Seema M, Bhawana D, Chitra S, Deepak G, Poodipedi Sarat C, Parthaprasad C. A combined gene signature of hypoxia and notch pathway in human glioblastoma and its prognostic relevance. PLoS One. 2015;10(3):e0118201.

6. Esposito CL, Nuzzo S, Catuogno S, Romano S, de Nigris F, de Franciscis V. STAT3 gene silencing by Aptamer-siRNA chimera as selective therapeutic for Glioblastoma. Mol Ther Nucleic Acids. 2018;10:398-411.

7. Masliantsev K, Pinel B, Balbous A, Guichet PO, Tachon G, Milin S, Godet J, Duchesne M, Berger A, Petropoulos C, et al. Impact of STAT3 phosphorylation in glioblastoma stem cells radiosensitization and patient outcome. Oncotarget. 2018;9(3):3968-79.

8. Liz J. Esteller M: IncRNAs and microRNAs with a role in cancer development. Biochim Biophys Acta. 2016;1859(1):169-76.

9. Evans JR, Feng FY, Chinnaiyan AM. The bright side of dark matter: IncRNAs in cancer. J Clin Investig. 2016;126(8):2775

10. Cao Y, Wang P, Ning S, Xiao W, Xiao B, Li X. Identification of prognostic biomarkers in glioblastoma using a long non-coding RNA-mediated, competitive endogenous RNA network. Oncotarget. 2016;7(27):41737-47.

11. Zhou M, Zhang Z, Zhao H, Bao S, Cheng L, Sun J. An immune-related sixIncRNA signature to improve prognosis prediction of Glioblastoma Multiforme. Mol Neurobiol. 2018;55(5):3684-97.

12. Tan SK, Pastori C, Penas C, Komotar RJ, Ivan ME, Wahlestedt C, Ayad NG. Serum long noncoding RNA HOTAIR as a novel diagnostic and prognostic biomarker in glioblastoma multiforme. Mol Cancer. 2018;17(1):74.

13. Bao ZS, Chen HM, Yang MY, Zhang CB, Yu K, Ye WL, Hu BQ, Yan W, Zhang W, Akers J. RNA-seq of 272 gliomas revealed a novel, recurrent PTPRZ1-MET fusion transcript in secondary glioblastomas. Genome Res. 2014;24(11): 1765-73.

14. Chaudhary K, Poirion OB, Lu L, Garmire LX, Chaudhary K, Poirion OB, Lu L, Garmire LX, Chaudhary K, Poirion OB. Deep Learning based multi-omics integration robustly predicts survival in liver cancer. Clin Cancer Res. 2017; 24(6):clincanres.0853.2017.

15. Wright MW. A short guide to long non-coding RNA gene nomenclature. Human genomics. 2014;8:7.

16. Wang L, Cao C, Ma Q, Zeng Q, Wang H, Cheng Z, Zhu G, Qi J, Ma H, Hai N. RNA-seq analyses of multiple meristems of soybean: novel and alternative transcripts, evolutionary and functional implications. BMC Plant Biol. 2014; 14(1):169 14,1(2014-06-17).

17. Hielscher T, Zucknick M, Werft W, Benner A. On the prognostic value of survival models with application to gene expression signatures. Stat Med. 2010;29(7-8):818-29.

18. Camp RL, Dolled-Filhart M, Rimm DL. X-tile: a new bio-informatics tool for biomarker assessment and outcome-based cut-point optimization. Clin Cancer Res. 2004;10(21):7252-9.

19. Eng KH, Emily S, Kayla M. On representing the prognostic value of continuous gene expression biomarkers with the restricted mean survival curve. Oncotarget. 2015;6(34):36308-18.

20. Huang G, Zhao G, Xia J, Wei Y, Chen F, Chen J, Shi J. FGF2 and FAM201A affect the development of osteonecrosis of the femoral head after femoral neck fracture. Gene. 2018;652:39-47.

21. Network TC. Corrigendum: comprehensive genomic characterization defines human glioblastoma genes and core pathways. Nature. 2013;494(7438):506.

22. Xu S, Liu C, Ma Y, Ji HL, Li X. Potential Roles of Amiloride-Sensitive Sodium Channels in Cancer Development. Biomed Res Int. 2016;2016(2):1-6 2016,(2016-6-15).
23. Tian $Y$, Bresenitz P, Reska A, El Moussaoui L, Beier CP, Grunder S. Glioblastoma cancer stem cell lines express functional acid sensing ion channels ASIC1a and ASIC3. Sci Rep. 2017;7(1):13674.

24. Kapoor N, Bartoszewski R, Qadri YJ, Bebok Z, Bubien JK, Fuller CM, Benos DJ. Knockdown of ASIC1 and epithelial sodium channel subunits inhibits glioblastoma whole cell current and cell migration. J Biol Chem. 2009; 284(36):24526-41.

25. Li Z, Du L, Li C, Wu W. Human chorionic gonadotropin $\beta$ induces cell motility via ERK1/2 and MMP-2 activation in human glioblastoma U87MG cells. J Neuro-Oncol. 2013;111(3):237-44.

26. Swisshelm K, Macek R, Kubbies M. Role of claudins in tumorigenesis. Adv Drug Deliv Rev. 2005;57(6):919-28.

27. Karnati HK, Panigrahi M, Shaik NA, Greig NH, Bagadi SA, Kamal MA, Kapalavayi N. Down regulated expression of Claudin-1 and Claudin-5 and up regulation of beta-catenin: association with human glioma progression. CNS Neurol Disord Drug Targets. 2014;13(8):1413-26.

28. Oohashi T, Edamatsu M, Bekku Y, Carulli D. The hyaluronan and proteoglycan link proteins: Organizers of the brain extracellular matrix and key molecules for neuronal function and plasticity. Exp Neurol. 2015;274(Pt B):134-44.

29. Hosung S, Bin H, Viapiano MS. Reduced expression of the hyaluronan and proteoglycan link proteins in malignant gliomas. J Biol Chem. 2009;284(39): 26547-56.

30. Chivukula M, Dincer HE, Biller JA, Krouwer HG, Simon G, Shidham V. FNAB cytology of extra-cranial metastasis of glioblastoma multiforme may resemble a lung primary: a diagnostic pitfall. CytoJournal. 2005;2:9.

31. Terada T. Expression of Cytokeratins in Glioblastoma Multiforme. Pathol Oncol Res. 2015;21(3):817-9.

32. Zhu M, Liu C, Li S, Zhang S, Yao Q, Song Q. Sclerostin induced tumor growth, bone metastasis and osteolysis in breast cancer. Sci Rep. 2017;7(1):11399.

33. Mcdonald MM, Delgado-Calle J. Sclerostin: an emerging target for the treatment of Cancer-induced bone disease. Curr Osteoporos Rep. 2017; 15(Suppl):1-10.

34. Lo SH. Tensin. Int J Biochem Cell Biol. 2004;36(1):31-4.

35. Muharram G, Sahgal P, Korpela T, Defranceschi N, Kaukonen R, Clark K, Tulasne D, Carpén O, Ivaska J. Tensin-4-dependent MET stabilization is essential for survival and proliferation in carcinoma cells. Dev Cell. 2014; 29(4):421-36.

36. Sawazaki S, Oshima T, Sakamaki K, Aoyama T, Sato T, Shiozawa M, Yoshikawa T, Rino Y, Imada T, Masuda M. Clinical significance of Tensin 4 gene expression in patients with gastric Cancer. Vivo. 2017;31(6):1065.

37. Misono S, Seki N, Mizuno K, Yamada Y, Uchida A, Sanada H, Moriya S, Kikkawa N, Kumamoto T, Suetsugu T, et al. Molecular Pathogenesis of Gene Regulation by the miR-150 Duplex: miR-150-3p Regulates TNS4 in Lung Adenocarcinoma. Cancers. 2019;11(5)

38. Bratt T. Lipocalins and cancer. Biochim Biophys Acta. 2000;1482(1):318-26.

39. Zheng LT, Lee S, Yin GN, Mori K, Suk K. Down-regulation of lipocalin 2 contributes to chemoresistance in glioblastoma cells. J Neurochem. 2009; 111(5):1238-51.

40. Wei BO, Wang LE, Chao DU, Guozhang HU, Wang L, Jin Y, Kong D. Identification of differentially expressed genes regulated by transcription factors in glioblastomas by bioinformatics analysis. Mol Med Rep. 2015;11(4): 2548-54.

41. Lei B, Yu L, Jung TA, Deng Y, Qi S. Prospective Series of Nine Long Noncoding RNAs Associated with Survival of Patients with Glioblastoma. J Neurol Surg Part A Cent Eur Neurosurg. 2018;79(6):471-8.

42. Lee $Y$, Lee J-K, Ahn SH, Lee J, Nam D-H. WNT signaling in glioblastoma and therapeutic opportunities. Lab Investig. 2016;96(2):137.

43. Leclerc C, Haeich J, Aulestia FJ, Kilhoffer M-C, Miller AL, Neant I, Webb SE,

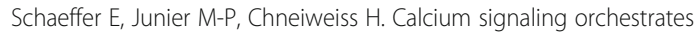
glioblastoma development: facts and conjunctures. Biochimica et Biophysica Acta (BBA)-Molecular. Cell Res. 2016;1863(6):1447-59.

44. Mazor G, Levin L, Picard D, Ahmadov U, Caren H, Borkhardt A, Reifenberger G, Leprivier G, Remke M, Rotblat B. The IncRNA TP73-AS1 is linked to aggressiveness in glioblastoma and promotes temozolomide resistance in glioblastoma cancer stem cells. Cell Death Dis. 2019;10(3):246.

45. Fu Q, Li S, Zhou Q, Yalikun K, Yisireyili D, Xia M. Low LINC00599 expression is a poor prognostic factor in glioma. Biosci Rep. 2019;39(4).

46. Xu CH, Xiao LM, Liu Y, Chen LK, Zheng SY, Zeng EM, Li DH. The IncRNA HOXA11-AS promotes glioma cell growth and metastasis by targeting miR130a-5p/HMGB2. Eur Rev Med Pharmacol Sci. 2019;23(1):241-52. 
47. Laks DR, Crisman TJ, Shih MY, Mottahedeh J, Gao F, Sperry J, Garrett MC, Yong WH, Cloughesy TF, Liau LM, et al. Large-scale assessment of the gliomasphere model system. Neuro-oncology. 2016;18(10):1367-78.

48. Chen X, Chen SI, Liu XA, Zhou WB, Ma RR, Chen L. Vav3 oncogene is upregulated and a poor prognostic factor in breast cancer patients. Oncol Lett. 2015;9(5):2143-8.

49. Zhong H, Zhong M. LINC00311 is overexpressed in ankylosing spondylitis and predict treatment outcomes and recurrence. BMC Musculoskelet Disord. 2019;20(1):278.

50. Wang Y, Luo TB, Liu L, Cui ZQ. LncRNA LINC00311 promotes the proliferation and differentiation of osteoclasts in osteoporotic rats through the notch signaling pathway by targeting DLL3. Cell Physiol Biochem. 2018; 47(6):2291-306.

\section{Publisher's Note}

Springer Nature remains neutral with regard to jurisdictional claims in published maps and institutional affiliations.

- fast, convenient online submission

- thorough peer review by experienced researchers in your field

- rapid publication on acceptance

- support for research data, including large and complex data types

- gold Open Access which fosters wider collaboration and increased citations

- maximum visibility for your research: over $100 \mathrm{M}$ website views per year

At $\mathrm{BMC}$, research is always in progress. 